\title{
La terapia de reemplazo hormonal disminuye la mortalidad en las mujeres
}

Grodstein F, Stampfer MJ, Colditz GA et al. Postmenopausal Hormone Therapy and Mortality. N Engl J Med 1997; 336:1769-75.

\section{Objetivo}

La terapia de reemplazo hormonal (TRH) tiene beneficios y riesgos: disminuye el riesgo de osteoporosis y enfermedad cardiovascular y aumenta el riesgo de cáncer de mama. El objetivo de este estudio es evaluar la relación entre mortalidad global y TRH, tanto en la población general de mujeres como en las mujeres con distintos factores de riesgo.

\section{Diseño}

Estudio caso-control anidado en una cohorte prospectiva. (Estudio de las Enfermeras).

\section{Lugar}

\section{Estados Unidos}

\section{Pacientes}

Enfermeras de 11 estados de EE.UU. Inicialmente 121.700 enfermeras entre 30 y 55 años de edad que contestaron un cuestionario por correo en 1976. Se repitieron los cuestionarios cada dos años hasta 1992. Se excluyeron del estudio pacientes que antes de la menopausia reportaran alguna enfermedad cardiovascular o cáncer, ya que esto podría influir en la prescripción de TRH luego de la menopausia. En este periodo hubo 3637 muertes que fueron los casos. Por cada mujer que falleció (caso) se seleccionaron al azar diez mujeres vivas al momento de su muerte (controles). Los controles eran similares a los casos en cuanto a edad, momento y tipo de menopausia.

\section{Evaluación de Factores de Riesgo}

Para cada fallecimiento se definió uso de TRH según el último cuestionario disponible antes de la muerte o diagnóstico de enfermedad fatal (esto reduce el sesgo causado por el abandono de la TRH luego del diagnóstico de una enfermedad potencialmente fatal).

\section{Medición de Resultados Principales}

El objetivo primario era medir la mortalidad total. Se obtuvo información del 98\% de las muertes (a través del certificado de defunción y en algunos casos la historia clínica). También se investigó mortalidad por enfermedad coronaria, enfermedad cerebro-vascular y cáncer.

\section{Resultados}

Luego de ajustar por distintas variables, quienes recibían TRH en ese momento tuvieron menor riesgo de muerte (riesgo relativo, 0.65 ; IC95\% 0.56-0.70) que las mujeres que nunca la recibieron. Este beneficio disminuía luego de más de 10 años de TRH (RR 0.80; IC95 0.67-0.96) debido a un aumento de la mortalidad por cáncer de mama en las tomadoras crónicas. Las pacientes con factores de riesgo coronario (69\%) obtuvieron la mayor reducción de mortalidad (RR 0.51; 0.45-0.57), mientras que las mujeres con bajo riesgo (13\%) no obtuvieron un beneficio significativo.

\section{Conclusiones}

La mortalidad promedio de las mujeres que reciben TRH es menor que las que no reciben, pero este beneficio disminuye a mayor duración de TRH y es menor en mujeres con bajo riesgo de enfermedad coronaria.

Apoyo Económico: National Institute of Health, USA
Los resultados proveen más reaseguro para la utilización combinada de estrógenos y progesterona ya que la reducción en la mortalidad fue observada también en este grupo de mujeres. La duda más importante que queda es si el grado de reducción de mortalidad es debido a las hormonas en sí o a que las pacientes que las reciben son más sanas, hipótesis sugerida por un estudio (4). El segundo estudio revisado es un análisis de decisión; una estrategia útil para combinar la evidencia de la literatura y estimar la expectativa de vida de distintas estrategias (en este caso dar o no dar TRH) en pacientes con distintos perfiles de riesgo. Todo análisis de decisión se basa en ciertas asunciones iniciales que tratan de ajustarse lo más posible a la realidad. En este caso ellos asumen que la cohorte que recibe TRH no difiere de la que no recibe en cuanto a la dieta, otras medicaciones o cuidados médicos, asunción poco realista como ya hemos visto. Se realizó un análisis de sen- sibilidad que comprueba la estabilidad de las conclusiones al modificar distintas variables. Ambos estudios sugieren un uso más amplio de la TRH desde el punto de vista de la expectativa de vida, aunque se plantean nuevos interrogantes como la duración óptima de la TRH y el momento ideal para iniciarla. Es importante considerar la actividad física como instrumento preventivo, no sólo por su valor preventivo de enfermedad coronaria y osteoporosis sino también de cáncer de mama, como muestra un estudio reciente revisado en este número de Evidencia (5). En resumen, para la decisión de administrar TRH importan tanto la actitud del médico y su actitud ante errores de acción (cáncer de mama) o de omisión (enfermedad coronaria y osteoporosis); como los perfiles de riesgo y las preferencias de nuestras pacientes.

Dr.Federico Augustovshi Unidad de Medicina Familiar y Preventiva Hospital Italiano de Buenos Aires

\section{REFERENCIAS}

1. Colditz GA, Manson JE, Hankinson SE. The Nurses' Health Study: 20-Year Contribution to the Understanding of Health Among Women. J of Women's Health 1997;6:49-62

2. Bergkvist L, Adami HO, Persson I et al. The risk of breast cancer afterestrogen and estrogen-progestin replacement. N Engl J Med 1989;321:293-7

3. Colditz GA, Hankinson SE, Hunter DJ et al. The use of estrogens and progestin and the risk of breast cancer in postmenopausal women. N Eng J Med 1995;332:1589-93

4. Matthews KA, Kuller LH, Wing RR et al. Prior to use of estrogen replacement therapy, are users healthier than nonusers? Am J Epidemiol 1996;143:971-8

5. Physical activity and the risk of breast cancer. N Engl J Med 1997; $336: 1269-1275$. 